\title{
Quantitative TIRF Microscopy for Counting Protein Copy Numbers in Isolated Sub-cellular Structures
}

\author{
Daniel T. Chiu*, Sarah A. Mutch*, Bryant Fujimoto*, Jennifer Gadd*, Sandra M. Bajjalieh** \\ * Department of Chemistry, University of Washington, Seattle, WA 98195. \\ ** Department of Pharmacology, University of Washington, Seattle, WA 98195.
}

We have recently developed a quantitative microscopy technique for quantifying the copy number of proteins in isolated sub-cellular structures. The method combines single-molecule totalinternal-reflection-fluorescence (TIRF) microscopy, microfluidics, and statistical de-convolution to ascertain the average number of proteins present on individual sub-cellular structures as well as the distribution in copy number about the average value [1].

Briefly, our approach relies on labeling sub-cellular structures, such as isolated sub-cellular organelles, with dye-tagged antibodies such that each protein is labeled with a single antibody. By determining the number of fluorescent antibodies attached to the sub-cellular structure or organelle, therefore, we can ascertain the number of a particular type of proteins present in the structure/organelle.

Because antibodies are dye labeled via free lysines, the number of fluorophores per antibody varies. As a result, we could not count the number of antibodies present simply from the measured fluorescence intensity level because a vesicle that bound two antibodies might exhibit higher fluorescence intensity than one with three antibodies. To de-convolve the variation in fluorescence intensity caused by differences in the number of dyes per antibody from the variation in intensity due to differences in the number of antibodies bound to vesicles, we compared the mean intensity and the shape of the intensity distribution of single-vesicle measurements to those obtained from single labeled antibodies. The measured intensities for both single antibodies and single vesicles show a lognormal distribution (see Figure 1). This statistical behavior indicates the source of our measured intensity spread is related in both the single-antibody and single-vesicle measurements, and that we could de-convolve the number of antibodies present on the vesicles simply by dividing the measured single-vesicle intensity distribution by a weighted linear combination of different scaled single-antibody intensity distribution. Unlike normal distributions, lognormal distributions have the property that the shape of the distribution does not change when multiplied by a scaling factor. To implement this de-convolution, we wrote a program that fits the single-vesicle intensity distribution with a weighted linear combination of single-antibody distributions that were generated by multiplying the measured single-antibody distribution by a given integer. Figure 2 shows the use of this technique for determining the number of dye-tagged biotins bound to single avidin molecules.

We have made a number of refinements to the TIRF microscope to enable quantitative microscopy measurements. We have also developed in-house software for both processing large numbers of images and for de-convolving the resulting intensity distributions. Finally, we applied this new technique to the quantification of the number of membrane proteins on synaptic vesicles.

References

[1] S.A. Mutch, B. Fujimoto, C.L. Kuyper, J.S. Kuo, S.M. Bajjalieh, D.T. Chiu (2007) "De-convolving single-molecule intensity distributions for quantitative microscopy measurements" Biophys. J. 92, 118.

[2] We gratefully acknowledge support from the National Institutes of Health (NS052637). 

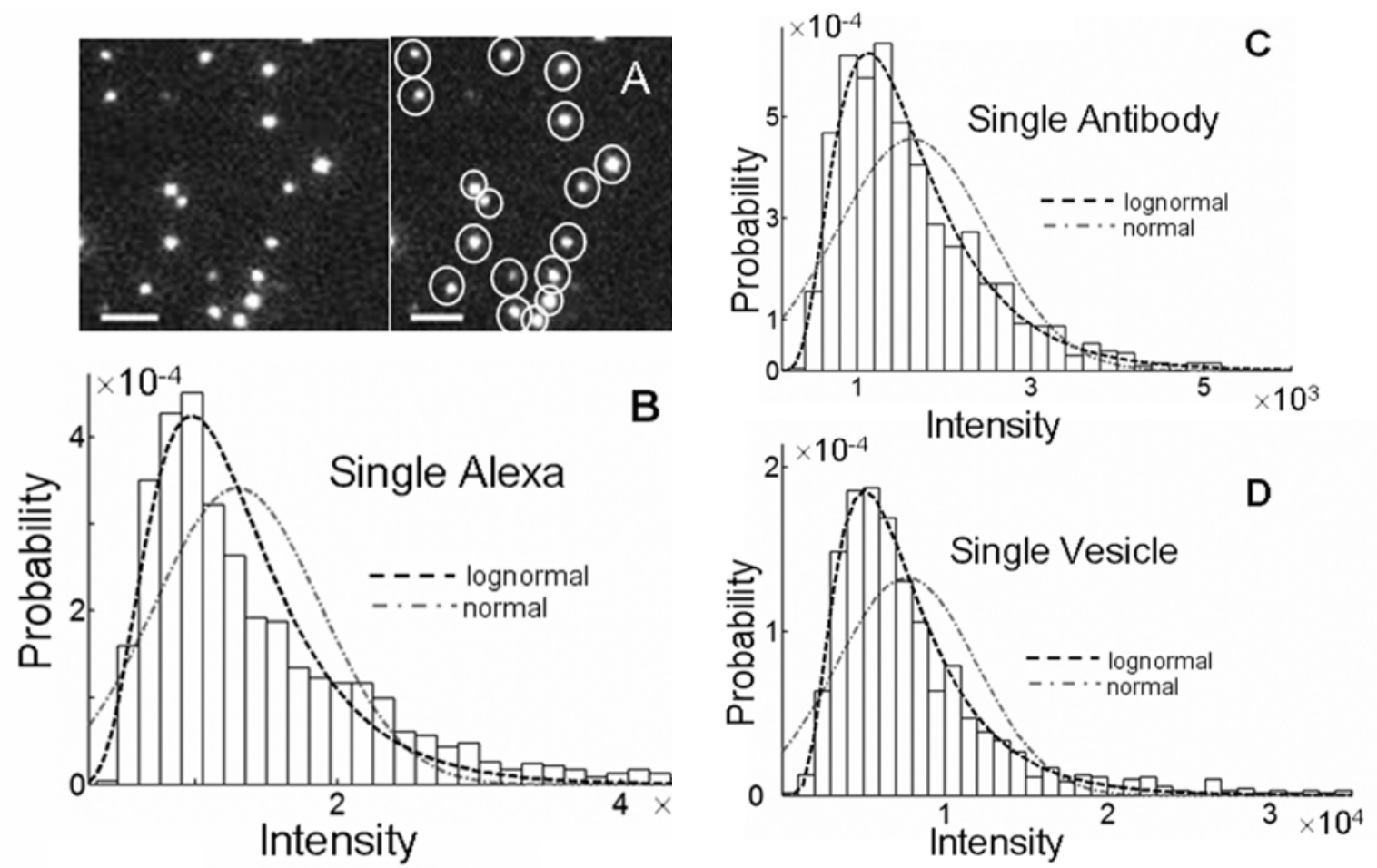

Figure 1. (A) Image of single goat anti-mouse (GAM) IgG antibodies labeled with multiple Alexa Fluor 488 dye molecules; the right panel shows each molecule being circled automatically by the imaging software. (B) A plot showing the fluorescence intensity distribution of single Alexa Fluor 488 molecules, (C) A plot showing the intensity distribution of single goat anti-mouse IgG molecules labeled with multiple Alexa Fluor 488, and (D) A plot showing the intensity distribution for single synaptic vesicles tagged with the anti-SV2 primary antibody and Alexa Fluor 488 labeled GAM secondary antibody.

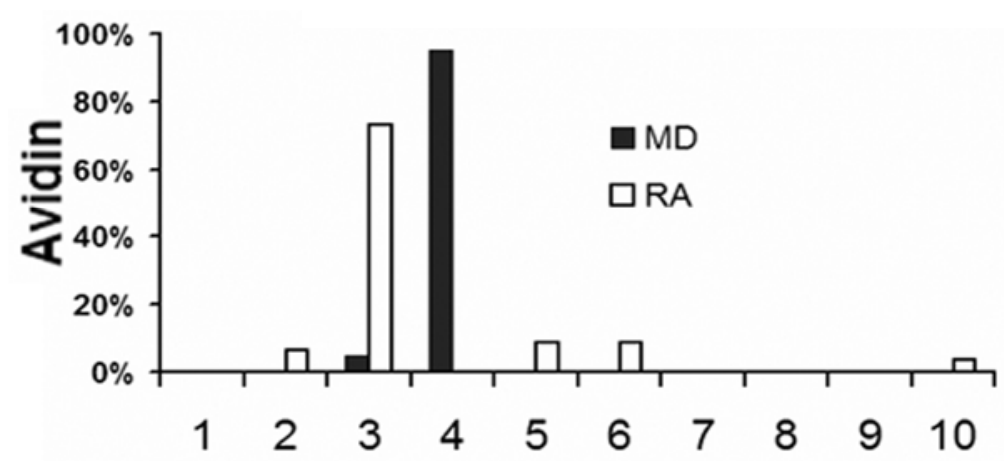

Figure 2. Histogram showing the percentage of avidin with c biocytins obtained from the fits using MD (dark vertical bars) and RA (light vertical bars) basis histograms. The use of the correct model (MD) gives the expected result (black bars in panel $\mathrm{C}$, which shows most avidins have 4 biocytins bound, which was also verified by our independent bulk measurements), but the use of the inappropriate model (RA) results in very different counts of the biocytins present (white bars in panel C), which for this model system are clearly incorrect. 